\title{
Recurrent, life-threatening atrioventricular dissociation associated with toxoplasma myocarditis
}

\author{
J S Duffield, A J Jacob, H C Miller
}

A 25 year old woman, on no medication and without significant medical history, developed symptoms of lethargy, fever, and muscle aches lasting 10 days, which resolved spontaneously.

One week later she became acutely unwell with dizziness, sweating, and chest tightness. On examination she had an irregular apical heart rate of 70 beats/minute. The blood pressure (BP) was initially $70 / 40 \mathrm{~mm} \mathrm{Hg}$, later becoming unrecordable. An electrocardiogram showed atrioventricular (AV) dissociation, ${ }^{1}$ with a nodal rate of 70 beats/minute and an atrial rate of 40 beats/minute. Intravenous atropine $(1 \mathrm{mg})$ induced rapid restoration of sinus rhythm and improvement in blood pressure and symptoms. Twelve hours later she remained well and in sinus rhythm. Cardiac enzymes and echocardiography were normal.

The following month she developed left cervical lymphadenopathy. Histology of an excised node showed evidence of infection with Toxoplasma gondii.

Soon after, she presented with a further episode of collapse associated with identical AV dissociation (figure), vasodilatation, and profound hypotension. Intravenous atropine ( $2 \mathrm{mg}$ ), isoprenaline, and two litres of intravenous colloid had no effect. The patient became drowsy, cold, clammy, and anuric. Echocardiography showed impaired left ventricular function. Infusion of dobutamine and then noradrenaline only raised the systolic BP to $60 \mathrm{~mm} \mathrm{Hg}$.

An atrial pacing wire was inserted. Wenckebach AV block occurred at an atrial pacing rate of 80 beats/minute. Dual chamber sequential pacing at 110 beats/minute restored $\mathrm{BP}$ to $100 / 50 \mathrm{~mm} \mathrm{Hg}$ with a brisk diuresis.

Twelve hours later, the jugular venous pressure was raised, a third heart sound was audible, the electrocardiogram showed widespread
$\mathrm{T}$-wave inversion, and LV function remained impaired on echocardiography. Atrial pacing alone induced Wenckebach at 100 beats/ minute, and 2:1 AV block at 120 beats/ minute. Ventricular pacing alone at 100 beats/minute induced symptoms and profound hypotension.

Thirty six hours later, all clinical and echocardiographic signs of LV impairment had resolved. The patient's rhythm had reverted to sinus rhythm and the BP was 120/80 mm Hg without inotropes. Protracted deliberate pacing of the atrium alone and then the ventricle alone produced normal responses. $\mathrm{T}$ wave inversion persisted in all leads, however. All serological tests were normal or negative except the IgM titre to Toxoplasma gondii, which was positive, and the Sabin-Feldman dye test, which was 1:500; together confirming active toxoplasmosis. Neither HIV serology nor myocardial biopsy was performed.

After a further identical presentation (except that now echocardiography and ECG were normal), requiring temporary dual chamber pacing, a course of pyrimethamine and spiromycin was given and a permanent dual chamber pacemaker was implanted. Despite the IgM titre and dye test suggesting persistent active infection, she remains well without symptoms one year later.

We believe this case is unique in that Toxoplasma gondii myocarditis resulted in recurrent, symptomatic AV dissociation with interference. ${ }^{1}$ During each attack an identical pattern of AV dissociation was present. Resolution of hypotension and symptoms followed AV sequential pacing. The patient was not bradycardic and LV dysfunction occurred only once. Thus AV dissociation caused the hypotension.

Rhythm strip showing sinus bradycardia at

40 beats/minute (arrows) with $A V$ dissociation from an idionodal escape rhythm. After the fourth $P$ wave, ventricular capture is seen. This rhythm was identical at each presentation.

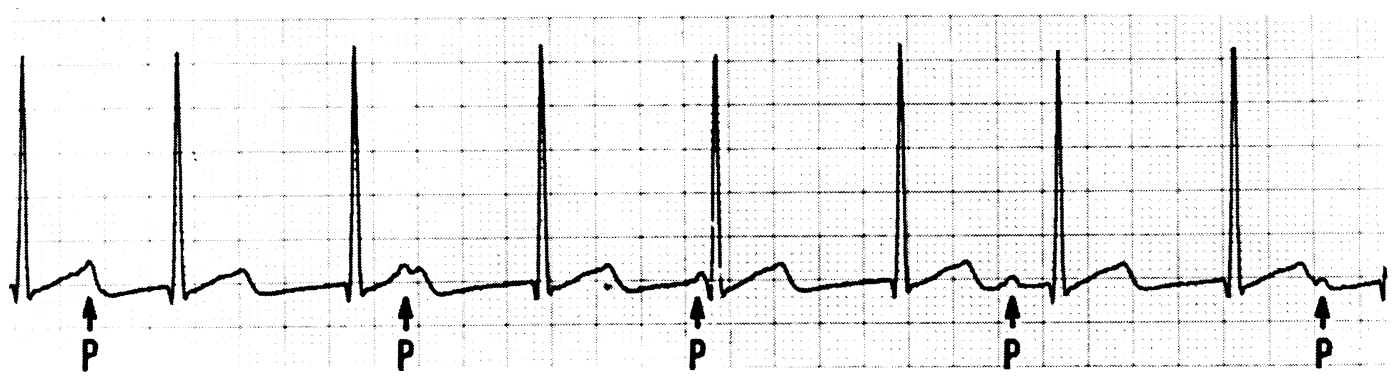


This pattern suggests that the patient was experiencing a condition similar to pacemaker syndrome, which occurs in some patients with a ventricular demand pacemaker. The severity of the condition is variable, ranging from flushing to hypotensive collapse, and is not always reproducible. There is often a delay of hours or days in the development of symptoms. ${ }^{2}$ The condition is thought to be mediated by several mechanisms including the activation of atrial stretch receptors during AV dissociation, causing an inappropriate reduction in peripheral resistance, ${ }^{3}$ and retrograde
VA conduction. The latter does not occur during $\mathrm{AV}$ dissociation with interference. However, VA conduction is not a prerequisite for development of pacemaker syndrome. ${ }^{2}$

We thank Dr Terry $\mathrm{H}$ Pringle, Consultant Cardiologist, Ninewells Hospital, Dundee, for assistance in compiling the clinical details.

1 Schott A. Atrioventricular dissociation with and without interference. Prog Cardiovasc Dis 1959;2:444. Ausubel K, Furman S. The pacemaker syndrome. Ann Intern Med 1985:103:420-9.

3 Travill CM, Sutton R. Pacemaker syndrome: an iatrogenic condition. Br Heart f 1992;68:163-6. 